\title{
PERSONALIDAD DE MARCA EN CARRERAS DE PSICOLOGÍA DE ZONAS TERRITORIALES EXTREMAS: ARICA Y PUNTA ARENAS
}

\author{
MARIANELA DENEGRI*, DANIEL CABEZAS*, MARJORIE NOVOA*, \\ JENNIFER PERALTA** \& CLAUDIA ESTRADA****
}

\begin{abstract}
RESUMEN
El objetivo de la presente investigación fue identificar los rasgos de la Personalidad de Marca de carreras de Psicología ubicadas en universidades estatales de zonas geográficamente extremas de Chile y que integran la Red de Escuelas de Psicología del Consorcio de Universidades del Estado (CUE). Para ello se utilizó la Escala de Personalidad de Marca de Aaker (1997), la que fue aplicada a 244 estudiantes de las carreras participantes, 148 de la Universidad de Tarapacá (región de Arica y Parinacota) y 96 de la Universidad de Magallanes (región de Magallanes y la Antártica Chilena). Los datos fueron sometidos a análisis factorial, el que mostró la presencia de 5 factores compuestos de 32 rasgos totales, el análisis por carrera muestra rasgos compartidos así como la existencia de rasgos específicos por universidad los que constituyen una Personalidad de Marca definida para cada carrera. Se discuten los resultados considerando la importancia de la Personalidad de Marca para la identidad institucional y la relación de estos rasgos con la identidad específica del territorio en el que se insertan las casas de estudio.
\end{abstract}

PALABRAS CLAVE: personalidad de marca, universidades regionales y estatales, mercado.

\section{BRAND PERSONALITY IN PSYCHOLOGY DEGREES IN EXTREME TERRITORIAL AREAS: ARICA AND PUNTA ARENAS}

\begin{abstract}
The purpose of this research was to identify the traits of the brand personality of psychology degrees located in public universities in geographically extreme areas and that form the Network of Schools of Psychology, State University Consortium (CUE). We used the scale of Aaker Brand Personality (1997), which was applied to 244 students to 244 students of the participating degrees, 148 from the University of Tarapacá (Arica and Parinacota Region) and 96 of the University of Magallanes (Magallanes and Chilean Antarctica Region). The data was subject to factor analysis, which showed the presence of 5 factors composed of

* Universidad de La Frontera, Departamento de Psicología. Av. Francisco Salazar 01145, Temuco, Chile.marianela.denegri@ ufrontera.cl; dcabezas@ufro.cl; m.novoa01@ufromail.cl.

** Dirección de Investigación y Extensión Académica. Universidad de Tarapacá. General Velásquez 1775, Arica, Chile. jperalta@ uta.cl.

*** Escuela de Psicología, Universidad de Magallanes. Av. Bulnes 01855, Punta Arenas, Chile. claudia.estrada@umag.cl.
\end{abstract}


32 total traits. The analysis shows shared traits as well as the existence of specific traits for University which constitute a brand personality defined for each institution. Results are discussed considering the importance of Brand Personality to the institutional identity and the relationship of these traits with the specific identity of the territory in which were the houses of study.

KEY WORDS: brand personality, regional and state universities, higher education market.

\section{INTRODUCCIÓN}

Durante las últimas décadas, las universidades han debido abordar importantes cambios asociados a la masificación de la educación superior, a la importancia de la economía del conocimiento y a los lineamientos gubernamentales asociados a una economía globalizada. Esto ha llevado a que estas instituciones, deban adaptarse a un entorno de complejidad e incertidumbre creciente y a abordar temáticas corporativas asociadas a estrategias gerenciales, sistemas de control de calidad (Parker et al., 2005), una mirada de un estudiante como usuario (sujeto a derechos y deberes) y a una creciente expansión de la oferta y, por tanto, a la necesidad de competir. $\mathrm{Al}$ respecto, Brunner (2008), plantea la existencia de un mercado de consumidores de servicios educativos, con amplia libertad de elección y que habitualmente pagan por el servicio (principalmente en pregrado) ya sea directamente o a través de créditos o becas, lo que evidentemente impacta el tipo de relación que estos establecen con las instituciones donde realizan sus estudios. ${ }^{1}$

En Chile, se observa un escenario donde la expansión ha llevado a la existencia de una matrícula total de 526.583 estudiantes de pregrado repartidos en 57 Universidades (Consejo Nacional de Educación, 2010), todas de distinta índole y distinta calidad, y con una regulación bastante baja, tanto en la creación como en la calidad de sus programas y funcionamiento general. En el caso particular de la oferta de carreras de psicología, esta ha ido en considerable aumento, llegando a existir un total de 144 programas de formación en el área y una matrícula anual de 5000 vacantes, de las cuales sólo un $2.9 \%$ corresponde a universidades estatales (Juliá, 2006).

Considerando estos nuevos escenarios de ex-

1 Investigación financiada por el proyecto DI11-2007, Dirección de Investigación, Universidad de la Frontera y patrocinado por el proyecto MECESUP ULS 0601 y convenios de desempeño UFRO0901, UTA0901 y MAG0901. pansión y competitividad, para cualquier institución de educación superior resulta esencial poder diferenciarse entre la diversidad de opciones académicas existentes, y ser reconocida con un sello distintivo que le permita generar valor agregado asociado a sus valores, línea de desarrollo y formación académica. En este contexto, resulta primordial conocer las impresiones que los usuarios y miembros de cada organización poseen sobre la misma y con ello aportar a la construcción de una imagen corporativa que revista sentido y significado para los usuariosclientes (Denegri et al., 2009).

Diversas investigaciones a nivel internacional (Aaker, 1997; Saavedra et al., 2008) y nacional (Denegri et al., 2009) han determinado la importancia de producir información de diferenciación de marca en sectores más amplios, incluyendo crecientemente a servicios que no habían sido considerados habitualmente en este tipo de estudios, como es el caso de los servicios educativos en general y de la educación superior en particular. Sin embargo, el sistema de educación superior es un sector que cuenta con marcas aún poco desarrolladas en su proceso de relación con sus grupos objetivos (Chapleo, 2005) constituyendo un área además con poca evidencia desde la investigación. Un incremento en la potencia y posicionamiento de la marca en una institución educativa aumenta su habilidad para competir por los mejores estudiantes y afianzar su posición en el escenario de instituciones similares (Judson, et al 2009). Desde el punto de vista de los estudiantes, la marca y sus características asociadas se constituyen en una promesa de satisfacer sus expectativas y facilita la decisión de seleccionar a una institución o de mantenerse en ella (Gupta \& Singh, 2010; Gopalan, et al., 2008; Lowrie, 2007; Nicholls et al.,1995).

Desde la perspectiva de la semiótica, la marca está constituida por un conjunto de discursos que mantienen entre sí todos los sujetos (individuales o colectivos) que participan en su génesis y desarrollo; a su vez, dicho en forma metafórica: una marca es un "motor semiótico», cuyo combustible 
está integrado por nombres, colores, sonidos, conceptos, objetos, sueños, deseos, etc., que, si se ha hecho funcionar el motor adecuadamente, dan como resultado un mundo ordenado, estructurado e interpretable (Semprin, 1995). Tal circunstancia exigía desde un principio necesariamente superar en cuanto se pudiera las formidables dificultades que oponían las características naturales del territorio patagónico occidental que por entonces y dado el elevado grado de desconocimiento que se tenía sobre el mismo por la clase dirigente del país -en el convencimiento de que cualquier alternativa viable o posible debía darse por suelo chileno- no dejaba más opción que la vía marítima, considerada en su empleo más seguro y eficiente para la navegación. Esta, en general preveía el uso del océano Pacífico hasta la latitud del canal de Chacao (pensando en Valparaíso y otros puertos de la zona metropolitana centro-sur como Talcahuano y Corral) y desde allí hacia el sur y viceversa el sistema del golfo de Corcovado y canales patagónicos septentrionales, otra vez el Pacífico para acceder al golfo de Penas y traspuesto este, el de los canales patagónicos meridionales y estrecho de Magallanes para unir a los puertos intermedios (Puerto Montt, Ancud, Castro) con Punta Arenas.

Pero en ese extenso tracto de mil millas el golfo de Penas conformaba por sus conocidas características de espacio marítimo abierto a las influencias oceánicas, el sector más riesgoso cuya navegación exigía el mayor cuidado de capitanes y pilotos de los barcos que debían seguirlo con sentido norte-sur y viceversa. Ello, naturalmente sin dejar de lado el progresivo mejor conocimiento de la hidrografía de la extensa ruta, materia en la que se estaba, casi sin pausa, desde los años de 1830 que cerraron el ciclo exploratorio emprendido por el Almirantazgo Británico a contar de 1826, en procura de la seguridad de la navegación y del comercio entre Europa y Sudamérica que en buena parte debía realizarse por la vía marítima franca como es el estrecho de Magallanes en el extremo meridional de América. Esta tarea había sido responsablemente asumida por la Armada de Chile a contar de los años de 1870 , entendida como una empresa laboriosa sin fecha de término. Las marcas se transforman entonces en el vehículo de la diferenciación, dada su capacidad de comunicar distintos tipos de significados y contribuyen fuertemente a la construcción de una identidad corporativa que refleje los valores que identifican a la organización y con ello aportan al compromiso de todos sus miembros (Lane \& Richey, 2006).

Uno de los aspectos centrales que constituyen a la marca es el concepto de Personalidad de Marca, entendido como un set de características asociadas a la Marca (Aaker, 1997), las que no sólo conllevan una serie de percepciones, asociaciones, recuerdos y prejuicios que el consumidor genera haciendo una imagen mental de sus ventajas y beneficios (Mercado, 2004), sino que también crean en el individuo un vínculo simbólico de autoexpresión (Keller, 1993) e identificación con el bien o servicio portador de la marca (Aaker, 1997). Este proceso se basa en atribuir características humanas y rasgos de personalidad a las marcas, proyectando así rasgos propios, ya sea por similitud ("esta marca se parece a mí") o posesión ("esta es mi marca") (Denegri et al., 2009). Se genera así una dinámica que lleva al usuario a asociarse emocionalmente con ella, que le imprime el status deseado dentro de su grupo cultural agregándole significado como socios de una relación cercana (Caprara et al., 2001, citado en Colmenares \& Saavedra, 2008; Denegri et al., 2009). Desde la perspectiva de la psicología social, la personalidad de marca puede ser entendida como un auto-estereotipo, es decir, una imagen mental de las características compartidas por un determinado grupo social al que se pertenece. En este sentido, la personalidad de marca es parte de la identidad colectiva de los miembros de una categoría puesto que visibiliza el prototipo que se establece como la mejor representación de cada uno de sus miembros (Hogg, 1996). La identidad colectiva es considerada como una parte del autoconcepto por lo que tiene efectos emocionales y conductuales tanto a nivel individual como grupal (Sedikides \& Brewer, 2001)

Diversas investigaciones han mostrado que esta capacidad de una marca para atraer a los consumidores mediante una combinación de características humanas asociadas a ellas, es un medio eficaz para distinguirla de sus competidores (Biel, 1993; Plummer, 1985; Yongjun \& Jooyoung, 2010; Freling et al., 2010). La personalidad de marca proporciona así una base para la diferenciación de sus servicios y cuando ésta es bien definida, conlleva una serie de beneficios y resultados enfocados en las preferencias ante su uso y favoritismo; aumento de los niveles de confianza y la lealtad; y creación de 
lazos emocionales (Fournier en Siguaw et al., 1999; Aaker, 1992). Así, la personalidad de la marca tiene dimensiones que reflejan tanto una percepción a nivel cognitivo como también fuertes componentes afectivos a la vez que su personificación incluye no sólo la atribución de características de personalidad y género sino también una asociación geográfica y territorial (Denegri, 2007).

Para medir Personalidad de Marca, existen una serie de métodos pero uno de los más utilizados y conocidos es el desarrollado por Aaker a partir de una serie de estudios empíricos realizados en Estados Unidos. Desde su publicación, el modelo de Aaker (1997) ha sido replicado y adaptado en diversos países en todo el mundo y trabajado por un gran número de investigadores (Koebel \& Ladwein, 1999; Aaker et al., 2001; Álvarez-Ortiz \& Harris, 2002; Supphellen \& Grohaug, 2003; Barrios \& Massa, 1999; Pirela et al, .2004; Saavedra et al, .2007; Yongjun \& Jooyoung, 2010; Freling et al., 2010). El modelo mencionado consta de cinco dimensiones principales de personalidad en las marcas, cada una de las cuales está conformada por una serie de rasgos distintivos.

En Chile, debido a su distribución geográfica y la gran diversidad cultural que esto genera, las instituciones de educación superior han considerado como parte de su propia definición institucional,las características de su entorno, y particularmente a las identidades y configuraciones propias de cada territorio. La identidad regional, al igual que el concepto de identidad, es dinámico y se refiere al proceso a través del cual las personas identifican un territorio junto con su cultura, tradiciones, paisaje, historia, entre otros (Atmann, 1997). La identidad regional se puede manifestar de diversas formas, como complejo de inferioridad o como orgullo, como sentimiento de pertenencia, derecho y proactividad cultural o política (Larraín, 2005). Asimismo, la identidad regional no puede entenderse sólo en términos del pasado o presente, sino también en términos de futuro, de las proyecciones, arquetipos, proyectos, mitos o metáforas con que la gente de una región o de un país se identifica en metas, horizontes de expectativas o utopías (SUBDERE, 2009). El desarrollo de la identidad regional se constituye en un proceso mediante el cual las personas reconocen un territorio y se identifican con él. Para Boisier (2006) esta identificación está fundada en creencias, continuidad histórica, actividades compartidas, un espacio y un sentido de pertenencia a partir de características socioculturales compartidas que le dan especificidad a una región dentro del contexto territorial e identitario nacional. El territorio aparece así como un elemento constitutivo de la identidad. Es aquello donde la identidad individual ancla su lugar de expresión y fija sus límites. Los individuos y los grupos existen a través de vivencias de territorialización múltiple. El concepto de identidad está cargado de territorialidad, de lugar propio, de espacio y de pertenencia.

Estos rasgos propios de los territorios, se representan en los habitantes de cada región como parte constitutiva de su propia identidad regional, que de acuerdo a Zúñiga \& Asún puede ser definida como aquella parte del autoconcepto de un individuo que está basada en su pertenencia a un grupo regional, junto con el significado valorativo y emocional asociado a dicha pertenencia (Zúñiga \& Asún, 2003: 75). Estos rasgos no sólo impactan a los individuos sino también a las instituciones, como es el caso de las universidades regionales, viéndose reflejadas en sus proyectos institucionales, y en sus objetivos de desarrollo, tanto por el impacto que ello tiene en su oferta académica como en la propia imagen corporativa. Ello es especialmente relevante en el caso de las universidades regionales ubicadas en zonas territoriales con claras características distintivas como es el caso de las regiones extremas de Arica y Parinacota en el norte y Magallanes y Antártica Chilena en el sur de nuestro país.

Las estadísticas indican que en Arica y Parinacota el $78.2 \%$ de la población considera que sí existe identidad regional; en tanto, para Magallanes la cifra aumenta a $82.6 \%$. Por otro lado, Magallanes está dentro de las regiones en que prima el imaginario de identidad regional por sobre la nacional, caso contrario para Arica y Parinacota en la que proclaman una identidad nacional por sobre las demás (SUBDERE, 2009, 2010).

La identidad regional de Arica y Parinacota está marcada por su ubicación fronteriza en el extremo norte de Chile, limitando con Bolivia y Perú lo que resulta en un asentamiento territorial con múltiples influencias externas y una gran presencia multicultural en la dinámica social donde el constante contacto social y económico con países limítrofes genera procesos de reconocimiento de las identidades grupales diferenciadas (chilenos, ariqueños, extranjeros, etc.) (Herrera, 2011). La multiculturalidad es característica 
que define e identifica a la región lo que incide en una identidad abierta a influencias externas que adapta costumbres, usos, rituales e imaginarios sociales que provienen del entorno chileno y del espacio cultural transfronterizo (Podestá, 2004). En Arica, la ciudad más septentrional de Chile, es posible constatar una fuerte adhesión a la figura de la Patria chilena tanto en las expresiones discursivas de sus habitantes como en la ritualidad cívica que ensalza la chilenidad por sobre el regionalismo (Díaz, Galdamez \& Ruz, 2010). La construcción de la identidad ariqueño-chilena se ha sustentado en un proceso de diferenciación con un"otro" que habita más al norte y sobre la férrea lealtad a la patria y su soberanía (González, 1997), manifestándose en celebraciones y prácticas que dan cuenta de una chilenidad muchas veces exacerbada (Díaz, Díaz \& Pizarro, 2010).

En relación a la Región de Magallanes y la Antártica Chilena, cuya ubicación territorial al sur es la más extrema del país, varios estudios (CEUTA, 2009; Méndez, 2009; Herrera,2011) que los participantes de la región de Magallanes (XII) presentan mayores porcentajes en el reconocimiento de una identidad característica y mantienen una identificación mayor con la región. Con respecto a la "Identidad Magallánica" esta se presenta como una identidad sólida y bien definida entre sus habitantes, marcada por el sentimiento de autorreconocimiento y a la vez de diferenciación reconocible y distinguible entre los habitantes, quienes inclusive dotan a esta categoría social de un alto grado de esencialismo donde la concepción de ser magallánico conlleva una complejidad mayor al sólo hecho de habitar un lugar geográfico determinado. Así, el ser magallánico supondría una esencia particular que es transmitida generación a generación como si estuviese inscrita en sus genes. Y aunque esta esencia única puede nutrirse del intercambio con otras culturas debe ser protegida dado que podría verse amenazada por ellas (Estrada \& Avedaño, 2008). Por otra parte también destaca una auto descripción de ser valientes y sufridos y valores como la solidaridad, sencillez, honestidad y una alta valoración de la unidad familiar, entre otras (Martinic, 2009; Molina, 2011).

En relación a las universidades y estudiantes de ambas regiones, se observa que según el informe anual INDICES 2010 (CNED, 2010) la región de Arica y Parinacota tenía 9.385 estudiantes de pregrado, de los cuáles 1.435 correspondían a estudiantes de carreras de las ciencias sociales. Específicamente en cuanto a la carrera de Psicología, en la región es dictada por una universidad estatal (UTA) y dos universidades privadas (Portal Carreras Universitarias, 2013). Por su parte, la región de Magallanes y la Antártica Chilena presentaba 4.926 estudiantes de pregrado, y de estos 361 correspondían a estudiantes del área de las ciencias sociales, siendo sólo la UMAG quien dicta la carrera de psicología en la región (Portal Carreras Universitarias, 2013).

Por estas características propias de cada territorio y por el nivel de competitividad del sistema de educación superior, resulta esencial tener una formación profesional que responda a las necesidades de su entorno desde sus propias identidades y que contribuya al desarrollo social, el cual constituye hoy día una misión esencial de la Educación Superior Contemporánea (UNESCO, 1998, en González, 2002).

A partir de estos antecedentes, el presente estudio se enmarca dentro de un macro-estudio en carreras de psicología de universidades del estado de Chile, cuyo propósito fue identificar las características y competencias que debían constituir el perfil de egreso de los futuros psicólogos formados en dichas casas de estudio y cuáles eran los rasgos propios de cada unidad educativa. (Proyecto MECESUP ULS 0602) para avanzar en la constitución de una identidad formativa propia de las universidades estatales. Ello resulta altamente relevante en un contexto donde existen más de 140 programas de psicología en el país, de los cuales sólo 10 corresponden a universidades estatales (mifuturo.cl, 2012). En este escenario, resultan especialmente atractivas para su estudio las carreras de psicología de la Universidad de Tarapacá y de la Universidad de Magallanes por su ubicación territorial extrema y porque ambas declaran en su misión institucional una vinculación y compromiso explícito con el desarrollo de las regiones en que están insertas (páginas institucionales, 2013). Específicamente en el caso de las carreras de psicología, en ambas universidades estas declaran el compromiso explícito con la realidad regional

Dentro de ese contexto, el trabajo que se presenta a continuación tiene como objetivos a) determinar la Personalidad de Marca de las carreras de Psicología de universidades ubicadas en las zonas territorialmente extremas de Chile y que además integran la Red de Psicología del Consorcio de Universidades del Estado (CUE), tal es el caso de las Universidades de Tarapacá 
y la Universidad de Magallanes y b) identificar aspectos distintivos de personalidad de marca que pudieran reflejar algunos rasgos identitarios regionales propios de cada universidad.

\section{MÉTODO}

\section{Participantes}

Los participantes del estudio fueron estudiantes de distintos niveles de las carreras de psicología de ambas universidades alcanzando un total de 244 participantes: 148 de la Universidad de Tarapacá y 96 de la Universidad de Magallanes. Del total de la muestra un $48.3 \%$ corresponde a hombres $(n=118)$ y un $51.6 \%$ a mujeres $(n=126)$. El rango de edad fluctúa entre 18 y 34 años, con un promedio de 21.8 años. El tipo de muestreo fue estratificado con fijación simple con un error Tipo I máximo de 0.05 y una potencia de prueba de 0.90 .

Los estudiantes accedieron a participar de forma voluntaria, firmando un consentimiento informado donde accedían a ser parte de dicho estudio. Mismo caso para las autoridades de ambas carreras que firmaron un documento similar para tales fines.

\section{Instrumento}

Se utilizó para la recolección de datos, la "Escala de personalidad de marca de Aaker" (1997). Esta posee originalmente 42 items agrupados en 5 factores (Sinceridad, Emocionante, Competencia, Sofisticación y Rudeza) que representan distintas dimensiones de personalidad de marca. Tiene un formato de respuesta tipo Likert, en escala de 1 a 5 según el grado de desacuerdo o acuerdo para cada ítem. Bajo el encabezado de a continuación, solicitamos su percepción acerca de una serie de características de la Carrera de Psicología de su casa de estudios, presentando una lista con todos los rasgos, los cuales debían ser encasillados de acuerdo al grado de acuerdo de representatividad que para el participante denota tal característica dentro de su carrera. Este instrumento cuenta con un índice de confiabilidad Alfa de Cronbach de 0,91 en mediciones con poblaciones universitarias del país (Denegri et al., 2009).

\section{Procedimiento}

En primera instancia, se contactaron las autoridades responsables de ambas carreras participantes, para solicitar su colaboración siendo informadas de los objetivos y relevancia de la presente investigación. A su vez, se firmó un consentimiento informado para sellar su conformidad.

Posteriormente, se llevó a cabo la aplicación por parte del equipo de investigación, del instrumento a los estudiantes en horarios regulares de clase, con un tiempo estimado de 15 minutos. Se solicitó la participación voluntaria, se dieron a conocer los objetivos y alcances de su participación, obteniendo su consentimiento informado y asegurando condiciones de confidencialidad.

\section{Plan de análisis}

Para el análisis de datos se utilizó el paquete estadístico SPSS v.17. Se realizó un análisis de validez factorial mediante el método de componentes principales con rotación ortogonal Varimax con Kayser. Siguiendo las recomendaciones de Nunnally (1978), Nunnally \& Bernstein (1994) y de Morales (2010), se seleccionó este método considerando la cantidad de ítems de la escala y que en todos los estudios previos se mostró una estructura factorial clara de 5 componentes.

Una vez establecida la distribución factorial, se utilizaron estadísticos descriptivos para conocer las medias de las variables, pruebas de confiabilidad y pruebas de muestras independientes para comparar a ambas instituciones con relación a los factores y las variables que conforman la personalidad de marca percibida entre los distintos niveles educativos, así como para realizar comparaciones de género en cada carrera. Una vez analizados los resultados, se realizó un análisis cualitativo de comparación de los rasgos más relevantes en cada carrera con aquellos que la literatura define como rasgos identitarios de cada región (Molina, 2011; Díaz, Galdámez et al., 2010; Díaz, Díaz \& Pizarro, 2010; Martinic, 2009; Estrada \& Avendaño, 2008)

\section{RESULTADOS}

En primer lugar, se calculó el índice en KaiserMeyer-Olkin (KMO = 0,886) y el test de esfericidad de Bartlett ( $B=3188,719 ; p<0,0001)$,los que mostraron una adecuación de los datos para el análisis propuesto.

Como ya fue señalado, el análisis factorial se realizó con el modelo de extracción de componentes principales y rotación Varimax con Kaiser, orientado a verificar la estructura factorial de la escala. Para la 
Tabla 1. Matriz de componentes rotados.

\begin{tabular}{|c|c|c|c|c|c|}
\hline & \multicolumn{5}{|c|}{ Componente } \\
\hline & 1 & 2 & 3 & 4 & 5 \\
\hline & Competencia & Sinceridad & Emocionante & Familiaridad & Robustez \\
\hline Confiada en si misma & 0,713 & & & & \\
\hline Competente & 0,698 & & & & \\
\hline Integra & 0,600 & & & & \\
\hline Moderna & 0,588 & & & & \\
\hline Inteligente & 0,572 & & & & \\
\hline Imaginativa & 0,555 & & & & \\
\hline Contemporánea & 0,538 & & & & \\
\hline Independiente & 0,500 & & & & \\
\hline Líder & 0,478 & & & & \\
\hline Colaborativa & 0,423 & & & & \\
\hline Sincera & & 0,775 & & & \\
\hline Honesta & & 0,725 & & & \\
\hline Confiable & & 0,719 & & & \\
\hline Segura & & 0,613 & & & \\
\hline Práctica & & 0,550 & & & \\
\hline Realista & & 0,469 & & & \\
\hline Excitante & & & 0,675 & & \\
\hline Fascinante & & & 0,640 & & \\
\hline Profunda & & & 0,614 & & \\
\hline Deslumbrante & & & 0,600 & & \\
\hline Original & & & 0,598 & & \\
\hline Osada & & & 0,409 & & \\
\hline Familiar & & & & 0,684 & \\
\hline Alegre & & & & 0,614 & \\
\hline Libre & & & & 0,595 & \\
\hline Amistosa & & & & 0,592 & \\
\hline Sentimental & & & & 0,557 & \\
\hline Ruda & & & & & 0,676 \\
\hline Masculina & & & & & 0,647 \\
\hline Clase alta & & & & & 0,594 \\
\hline Obstinada & & & & & 0,577 \\
\hline Elitista & & & & & 0,537 \\
\hline
\end{tabular}

Método de extracción: Análisis de componentes principales.

Método de rotación: Normalización Varimax con Kaiser.

inclusión de los elementos correspondientes a cada factor se siguieron los siguientes criterios: a)Aquellos elementos con una saturación igual o superior a 4,0 en la matriz de componentes rotados; y b) se seleccionó la saturación de mayor valor en aquellos casos en que se daba en más de un factor. Con estos criterios se aislaron cinco factores con valor propio o igual a 1,00 que explicaba el $50,487 \%$ de la varianza total, los que en su totalidad incorporaron 32 de los 42 rasgos que originalmente consideraba la escala en sus versiones previas (Denegri et al., 2009).

A diferencia de la escala original de Aaker (1997), a pesar que emergen 5 factores, se observa una distribución de rasgos al interior de los mismos que constituye una nueva estructura. Así, se identifican los factores Competencia con 10 rasgos; Sinceridad con 6 rasgos; Emocionante o Excitante con 6 rasgos; Familiaridad con 5 rasgos y finalmente Rudeza o Robustez con 5 rasgos, como se muestra en la Tabla 1. El análisis de fiabilidad mediante Alpha de 
Tabla 2. Medias de factores por Universidad.

\begin{tabular}{|c|c|c|c|c|c|c|c|c|c|}
\hline & & \multirow{2}{*}{$\mathrm{N}$} & \multirow{2}{*}{ Media } & \multirow{2}{*}{$\begin{array}{c}\text { Desviación } \\
\text { típica }\end{array}$} & \multirow{2}{*}{$\begin{array}{l}\text { Error } \\
\text { típico }\end{array}$} & \multicolumn{2}{|c|}{$\begin{array}{l}\text { Intervalo de confianza } \\
\text { para la media al } 95 \%\end{array}$} & \multirow{2}{*}{ Mínimo } & \multirow{2}{*}{ Máximo } \\
\hline & & & & & & $\begin{array}{l}\text { Limite } \\
\text { inferior }\end{array}$ & $\begin{array}{l}\text { Limite } \\
\text { superior }\end{array}$ & & \\
\hline \multirow{3}{*}{ Competencia } & UMAG & 96 & 3,5896 & 0,59232 & 0,06045 & 3,4696 & 3,7096 & 2,00 & 5,00 \\
\hline & UTA Arica & 134 & 3,5291 & 0,65245 & 0,05636 & 3,4176 & 3,6406 & 1,30 & 4,90 \\
\hline & Total & 230 & 3,5543 & 0,62743 & 0,04137 & 3,4728 & 3,6359 & 1,30 & 5,00 \\
\hline \multirow{3}{*}{ Sinceridad } & UMAG & 96 & 3,5330 & 0,72290 & 0,07378 & 3,3865 & 3,6795 & 1,17 & 4,83 \\
\hline & UTA Arica & 140 & 3,3929 & 0,74912 & 0,06331 & 3,2677 & 3,5180 & 1,50 & 5,00 \\
\hline & Total & 236 & 3,4499 & 0,74024 & 0,04819 & 3,3549 & 3,5448 & 1,17 & 5,00 \\
\hline \multirow{3}{*}{ Emocionante } & UMAG & 96 & 3,2049 & 0,71879 & 0,07336 & 3,0592 & 3,3505 & 1,17 & 4,67 \\
\hline & UTA Arica & 138 & 3,0906 & 0,73249 & 0,06235 & 2,9673 & 3,2139 & 1,17 & 5,00 \\
\hline & Total & 234 & 3,1375 & 0,72753 & 0,04756 & 3,0438 & 3,2312 & 1,17 & 5,00 \\
\hline \multirow{3}{*}{ Familiaridad } & UMAG & 96 & 3,3917 & 0,69459 & 0,07089 & 3,2509 & 3,5324 & 1,60 & 5,00 \\
\hline & UTA Arica & 141 & 3,3348 & 0,77625 & 0,06537 & 3,2055 & 3,4640 & 1,00 & 5,00 \\
\hline & Total & 237 & 3,3578 & 0,74327 & 0,04828 & 3,2627 & 3,4529 & 1,00 & 5,00 \\
\hline \multirow[t]{3}{*}{ Rudeza } & UMAG & 96 & 2,4167 & 0,60640 & 0,06189 & 2,2938 & 2,5395 & 1,20 & 4,00 \\
\hline & UTA Arica & 137 & 2,3766 & 0,70630 & 0,06034 & 2,2573 & 2,4960 & 1,00 & 5,00 \\
\hline & Total & 233 & 2,3931 & 0,66588 & 0,04362 & 2,3072 & 2,4791 & 1,00 & 5,00 \\
\hline
\end{tabular}

Cronbach indica que tanto la escala total como cada uno de los factores presentan valores adecuados. Se aprecia así un Alpha de 0.901 para la escala total y para cada uno de los factores arroja: Competencia 0.856; Sinceridad 0.836; Emocionante 0.792; Familiaridad 0.775 y finalmente para el factor Rudeza un alpha de 0.726 .

En relación al análisis comparativo entre las carreras de cada universidad para cada uno de los factores, se aplicó una prueba t de Student para muestras independientes. Los resultados indican que no se observan diferencias significativas lo que indica que los factores se comportan de manera homogénea en ambas muestras. En términos específicos, para el Factor Competencia se observa una $\mathrm{t}=0,732$, gl $215 p=0,472$; para el Factor Confiabilidad una $\mathrm{t}=$ 1,432,gl 234 p = 0,154; para el Factor Emocionante $\mathrm{t}=1,183, \mathrm{gl} 232, \mathrm{p}=0,238$; para el Factor Familiaridad $\mathrm{t}=0,578, \mathrm{gl} 235, \mathrm{p}=0,564 \mathrm{y}$ finalmente para el Factor Rudeza se aprecia una $t=0,451$, gl 231, $p=0,653$.

Para el análisis de medias de los factores para cada carrera, se estableció como factor relevante aquel con una media superior a la media métrica teórica de la escala $(3,0)$. Para la carrera de Psicología de la Universidad de Tarapacá (UTA),el factor Competencia $(3,52)$ es el que presenta la media más alta seguido del factor Confiabilidad (3,39), Familiaridad $(3,33)$, Emocionante $(3,09)$. En el caso de la carrera de
Psicología de la Universidad de Magallanes (UMAG) las medias más altas corresponden a los factores Competencia $(3,58)$ y Confiabilidad $(3,53)$, seguidos de los factores Familiaridad $(3,39)$ y Emocionante $(3,20)$. En ambas universidades, el factor Rudeza es el que presenta las medias más bajas (UTA 2,37 y UMAG 2,41) (Ver Tabla 2)

En cuanto al análisis por rasgos sobresalientes en cada factor para cada una de las universidades, se determinó como rasgos relevante aquellos cuya media era superior a la media métrica teórica de la escala $(3,0)$.

Los resultados arrojados, nos indican que para el caso de la Carrera de Psicología de la UTA de un total de 32 rasgos, 15 de éstos se ubican en un rango sobre 3,0, por lo que son percibidas como distintivas de la carrera, entre los cuales destacan rasgos como Competente $(\mathrm{M}=3,87)$, Inteligente $(\mathrm{M}=3,87)$, Colaborativa (3,70), Amistosa (3,70), Alegre $(3,56)$, Realista (3,58), Confiada en si misma $(3,52)$ Práctica $(3,51)$ y Segura $(3,51)$. (Ver Tabla 3).

Para el caso de la Carrera de Psicología de la UMAG, de un total de 32 rasgos medidas por la Escala de Personalidad de Marca, la media de 22 de ellos se ubican en un rango sobre 3,0, por lo que son percibidas como distintivas de la carrera. Destacan rasgos como Competente $(3,88)$, Confiable $(3,79)$, Amistosa (3,76)Osada (3,75), Realista $(3,74)$ e Ima- 
Tabla 3. Rasgos característicos (Universidad de Tarapacá).

\begin{tabular}{lccc}
\hline Rasgos & Media & $\begin{array}{c}\text { Desviación } \\
\text { típica }\end{array}$ & Factor \\
\hline Competente & 3,87 & 0,940 & Competencia \\
Inteligente & 3,87 & 0,871 & Competencia \\
Colaborativa & 3,70 & 0,951 & Competencia \\
Amistosa & 3,70 & 0,961 & Familiaridad \\
Alegre & 3,56 & 0,956 & Familiaridad \\
Realista & 3,58 & 0,935 & Sinceridad \\
Confiada en si & 3,52 & 0,933 & Competencia \\
misma & 3,52 & 0,990 & Competencia \\
Integra & 3,51 & 1,058 & Sinceridad \\
Práctica & 3,51 & 1,002 & Sinceridad \\
Segura & 3,49 & 1,001 & Sinceridad \\
Confiable & 3,48 & 1,003 & Competencia \\
Contemporánea & 3,38 & 0,835 & Emocionante \\
Profunda & 3,32 & 0,845 & Competencia \\
Moderna & 3,32 & 0,825 & Emocionante \\
Original & & &
\end{tabular}

ginativa (3,74). (Ver Tabla 4).

Se observan 15 rasgos comunes entre ambas universidades (alegre, amistosa, colaborativa, competente, confiable, confiada en sí misma, contemporánea, integra, inteligente, moderna, original, práctica, profunda, realista, segura) observándose diferencias significativas sólo en el rasgo confiable ( $\mathrm{t}=2,35$, gl 237, $\mathrm{p}=0.019)$ donde la media más alta la presenta la UMAG $(3,79)$ en comparación a la UTA $(3,49)$. En ambas universidades, la mayor cantidad de rasgos corresponden al factor Competencia.

Como se observa en la Tabla 5, en relación al análisis por género en la Universidad de Tarapacá, este arrojo a partir de la prueba de Levene que los grupos son homogéneos para todos los factores a analizar (Competencia: $\mathrm{F}=0,057, \mathrm{p}=0,812$; Confiabilidad: $F=2,926, p=0,089$; Emocionante: $F=$ 0,012, $p=0,914$; Familiaridad: $F=2,843, p=0,094$; Rudeza: $F=0,015, p=0,902)$, y que estos presentan diferencias significativas en los factores Sinceridad $(t=-1,974, p=0,050) y$ Rudeza $(t=1,960, p=0,052)$, y no así en los factores Competencia ( $t=-1,613, p=0$, 109), Emocionante ( $\mathrm{t}=-0,822, \mathrm{p}=0,412)$ y Familiaridad $(\mathrm{t}=-0,224, \mathrm{p}=0,823)$. En el Factor Sinceridad son las mujeres quienes presentan una media más alta que los hombres, mientras que en el Factor Rudeza son los hombres quienes presentan una media superior a la de las mujeres.

Para el caso de la Universidad de Magallanes, a partir de la prueba de Levene se asume que los grupos
Tabla 4. Rasgos característicos (Universidad de Magallanes).

\begin{tabular}{lccc}
\hline Rasgos & Media & $\begin{array}{c}\text { Desviación } \\
\text { típica }\end{array}$ & Factor \\
\hline Inteligente & 3,96 & 0,870 & Competencia \\
Competente & 3,88 & 0,976 & Competencia \\
Confiable & 3,79 & 0,882 & Sinceridad \\
Amistosa & 3,76 & 0,937 & Familiaridad \\
Osada & 3,75 & 0,929 & Emocionante \\
Realista & 3,74 & 0,811 & Sinceridad \\
Imaginativa & 3,74 & 0,837 & Competencia \\
Contemporánea & 3,69 & 0,825 & Competencia \\
Segura & 3,61 & 1,019 & Sinceridad \\
Integra & 3,61 & 0,826 & Competencia \\
Alegre & 3,61 & 0,826 & Familiaridad \\
Fascinante & 3,60 & 1,147 & Emocionante \\
Profunda & 3,58 & 0,981 & Emocionante \\
Colaborativa & 3,56 & 0,993 & Competencia \\
Honesta & 3,51 & 0,995 & Sinceridad \\
Independiente & 3,50 & 0,951 & Competencia \\
Confiada en sí & 3,50 & 0,984 & Competencia \\
misma & 3,31 & 0.987 & Competencia \\
Moderna & 3,29 & 1,080 & Familiaridad \\
Familiar & 3,28 & 0,879 & Sinceridad \\
Sincera & 3,26 & 1,126 & Sinceridad \\
Practica & 3,26 & 0,824 & Emocionante \\
Original & & &
\end{tabular}

son homogéneos para todos los factores a analizar (Competencia: $\mathrm{F}=1,193, \mathrm{p}=0,278$; Confiabilidad: $\mathrm{F}=0,000, \mathrm{p}=0,989$; Emocionante: $\mathrm{F}=0,874, \mathrm{p}=$ 0,352; Familiaridad: $\mathrm{F}=0,367, \mathrm{p}=0,546$; Rudeza: $\mathrm{F}=$ $0,967, p=0,328)$, y que estos no presentan diferencias significativas para los factores analizados: Competencia $(t=-1,744, p=0,084)$, Rudeza $(t=0,848, p=0,399)$, Confiabilidad ( $\mathrm{t}=-0,140, \mathrm{p}=0,889)$, Emocionante $(\mathrm{t}=$ $1,121, p=0,904)$ y Familiaridad $(t=0,141, p=0,888)$. (Ver Tabla 6).

\section{DISCUSIÓN Y CONCLUSIONES}

La discusión la abordaremos a partir de los objetivos formulados para este estudio para luego reflexionar sobre algunos aspectos generales vinculados a la necesidad de diferenciación y posicionamiento a las que se enfrentan las carreras de nuestras instituciones de educación superior en un contexto global de incertidumbre y competitividad.

Como se analizó anteriormente, la situación actual de la educación superior en Chile muestra niveles de alta competitividad, que han estimulado 
Tabla 5. Medias Factores por Género y prueba de muestras independientes por Género - UTA.

\begin{tabular}{|c|c|c|c|c|c|c|c|c|c|c|c|c|}
\hline & & \multicolumn{2}{|c|}{ Género } & \multicolumn{2}{|c|}{$\begin{array}{c}\text { Prueba de } \\
\text { Levene para } \\
\text { la igualdad de } \\
\text { varianzas }\end{array}$} & \multicolumn{4}{|c|}{ Prueba T para la igualdad de medias } & \multirow{3}{*}{$\begin{array}{l}\text { Error } \\
\text { típ. de la } \\
\text { diferen. }\end{array}$} & \multirow{2}{*}{\multicolumn{2}{|c|}{$\begin{array}{l}\text { 95\% Intervalo de } \\
\text { confianza para la } \\
\text { diferencia }\end{array}$}} \\
\hline & & & & $\mathrm{F}$ & Sig. & $\mathrm{t}$ & $\mathrm{gl}$ & $\begin{array}{l}\text { Sig. } \\
\text { (bilat.) }\end{array}$ & $\begin{array}{l}\text { Difer. de } \\
\text { medias }\end{array}$ & & & \\
\hline & & $\begin{array}{l}\text { Media } \\
\text { Hombre }\end{array}$ & $\begin{array}{l}\text { Media } \\
\text { Mujer }\end{array}$ & & & & & & & & Inferior & Superior \\
\hline \multirow{2}{*}{ Competencia } & $\begin{array}{l}\text { Se han asumido } \\
\text { varianzas iguales }\end{array}$ & 3,4 & 3,59 & 0,057 & 0,812 & $-1,613$ & 130 & 0,109 & $-0,189$ & 0,117 & $-0,421$ & 0,043 \\
\hline & $\begin{array}{c}\text { No se han asumido } \\
\text { varianzas iguales }\end{array}$ & & & & & $-1,616$ & 98,439 & 0,109 & $-0,189$ & 0,117 & $-0,421$ & 0,043 \\
\hline \multirow{2}{*}{ Sinceridad } & $\begin{array}{l}\text { Se han asumido } \\
\text { varianzas iguales }\end{array}$ & 3,21 & 3,47 & 2,926 & 0,089 & $-1,974$ & 136 & 0,05 & $-0,259$ & 0,131 & $-0,518$ & 0,000 \\
\hline & $\begin{array}{l}\text { No se han asumido } \\
\text { varianzas iguales }\end{array}$ & & & & & $-1,865$ & 81,979 & 0,066 & $-0,259$ & 0,139 & $-0,535$ & 0,017 \\
\hline \multirow{2}{*}{ Emocionante } & $\begin{array}{l}\text { Se han asumido } \\
\text { varianzas iguales }\end{array}$ & 3,02 & 3,12 & 0,012 & 0,914 & $-0,822$ & 135 & 0,412 & $-0,108$ & 0,132 & $-0,369$ & 0,152 \\
\hline & $\begin{array}{c}\text { No se han asumido } \\
\text { varianzas iguales }\end{array}$ & & & & & $-0,82$ & 95,793 & 0,414 & $-0,108$ & 0,132 & $-0,371$ & 0,154 \\
\hline \multirow{2}{*}{ Familiaridad } & $\begin{array}{l}\text { Se han asumido } \\
\text { varianzas iguales }\end{array}$ & 3,31 & 3,34 & 2,843 & 0,094 & $-0,224$ & 138 & 0,823 & $-0,031$ & 0,139 & $-0,306$ & 0,244 \\
\hline & $\begin{array}{l}\text { No se han asumido } \\
\text { varianzas iguales }\end{array}$ & & & & & $-0,215$ & 85,783 & 0,83 & $-0,031$ & 0,145 & $-0,319$ & 0,256 \\
\hline \multirow{2}{*}{ Rudeza } & $\begin{array}{l}\text { Se han asumido } \\
\text { varianzas iguales }\end{array}$ & 2,54 & 2,29 & 0,015 & 0,902 & 1,96 & 133 & 0,052 & 0,247 & 0,126 & $-0,002$ & 0,497 \\
\hline & $\begin{array}{l}\text { No se han asumido } \\
\text { varianzas iguales }\end{array}$ & & & & & 1,896 & 88,021 & 0,061 & 0,247 & 0,131 & $-0,012$ & 0,507 \\
\hline
\end{tabular}

crecientes esfuerzos de las distintas instituciones por posicionarse en un contexto de aumento progresivo de la oferta. Ello es especialmente evidente en el explosivo crecimiento que ha manifestado la matrícula en la carrera de psicología, con escasa presencia en ella de las universidades del consorcio de universidades estatales (CUE), las cuáles han privilegiado la formación de calidad en lugar de la masificación de la formación profesional (Juliá, 2006). Frente a esta decisión, resulta estratégico poder contar con evidencia acerca de la percepción de los estudiantes como usuarios directos del servicio educativo, de forma de verificar hasta dónde esta opción se refleja efectivamente en las características que estos atribuyen a la carrera que cursan.

Desde esta perspectiva, se esperaría que dentro de los rasgos percibidos como propios de la perso- nalidad de marca de sus carreras, tuvieran especial relevancia aquellos vinculados a la Competencia en el ejercicio formativo y la Sinceridad que se relaciona con la credibilidad y confiabilidad. Los resultados muestran efectivamente que el factor Competencia es el que muestra la media más alta $(3,55)$ seguido por Sinceridad $(3,44)$, sin diferencias entre ambas universidades. Este resultado es coincidente con otros estudios realizados también en carreras de psicología del CUE que manifiestan la misma tendencia y que por tanto reafirman la decisión de optar por calidad en lugar de aumento expansivo de matrículas(Denegri et al., 2009).

Otro aspecto interesante que emerge del análisis es la presencia de rasgos característicos para cada una de las universidades, de los cuales 15 son compartidos entre ambas instituciones sin diferencias 
Tabla 6. Medias Factores por Género y Prueba de muestras independientes por Género - UMAG.

\begin{tabular}{|c|c|c|c|c|c|c|c|c|c|c|c|c|}
\hline & & \multicolumn{2}{|c|}{ Género } & \multicolumn{2}{|c|}{$\begin{array}{c}\text { Prueba de } \\
\text { Levene para } \\
\text { la igualdad de } \\
\text { varianzas }\end{array}$} & \multicolumn{4}{|c|}{ Prueba $T$ para la igualdad de medias } & \multirow{3}{*}{$\begin{array}{l}\text { Error típ. } \\
\text { Difer. }\end{array}$} & \multirow{2}{*}{\multicolumn{2}{|c|}{$\begin{array}{c}\text { 95\% Intervalo de } \\
\text { confianza para la } \\
\text { diferencia }\end{array}$}} \\
\hline & & & & $\mathrm{F}$ & Sig. & $\mathrm{t}$ & $\mathrm{gl}$ & $\begin{array}{l}\text { Sig. } \\
\text { (bilat.) }\end{array}$ & $\begin{array}{l}\text { Diferencia } \\
\text { de medias }\end{array}$ & & & \\
\hline & & $\begin{array}{l}\text { Media } \\
\text { Hombre }\end{array}$ & $\begin{array}{l}\text { Media } \\
\text { Mujer }\end{array}$ & & & & & & & & Inferior & Superior \\
\hline \multirow{2}{*}{ Competencia } & $\begin{array}{l}\text { Se han asumido } \\
\text { varianzas iguales }\end{array}$ & 3,43 & 3,65 & 1,193 & 0,278 & $-1,744$ & 94 & 0,084 & $-0,227$ & 0,130 & $-0,486$ & 0,031 \\
\hline & $\begin{array}{c}\text { No se han asumido } \\
\text { varianzas iguales }\end{array}$ & & & & & $-1,719$ & 51,576 & 0,092 & $-0,227$ & 0,132 & $-0,492$ & 0,038 \\
\hline \multirow{2}{*}{ Sinceridad } & $\begin{array}{l}\text { Se han asumido } \\
\text { varianzas iguales }\end{array}$ & 3,51 & 3,53 & 0 & 0,989 & $-0,14$ & 94 & 0,889 & $-0,023$ & 0,162 & $-0,343$ & 0,298 \\
\hline & $\begin{array}{c}\text { No se han asumido } \\
\text { varianzas iguales }\end{array}$ & & & & & $-0,147$ & 60,54 & 0,883 & $-0,023$ & 0,153 & $-0,329$ & 0,284 \\
\hline \multirow{2}{*}{ Emocionante } & $\begin{array}{l}\text { Se han asumido } \\
\text { varianzas iguales }\end{array}$ & 3,21 & 3,19 & 0,874 & 0,352 & 0,121 & 94 & 0,904 & 0,019 & 0,161 & $-0,300$ & 0,338 \\
\hline & $\begin{array}{c}\text { No se han asumido } \\
\text { varianzas iguales }\end{array}$ & & & & & 0,131 & 64,949 & 0,896 & 0,019 & 0,148 & $-0,276$ & 0,315 \\
\hline \multirow{2}{*}{ Familiaridad } & $\begin{array}{l}\text { Se han asumido } \\
\text { varianzas iguales }\end{array}$ & 3,4 & 3,38 & 0,367 & 0,546 & 0,141 & 94 & 0,888 & 0,022 & 0,155 & $-0,286$ & 0,330 \\
\hline & $\begin{array}{c}\text { No se han asumido } \\
\text { varianzas iguales }\end{array}$ & & & & & 0,146 & 58,542 & 0,884 & 0,022 & 0,149 & $-0,277$ & 0,320 \\
\hline Rudeza & $\begin{array}{l}\text { Se han asumido } \\
\text { varianzas iguales }\end{array}$ & 2,49 & 2,38 & 0,967 & 0,328 & 0,848 & 94 & 0,399 & 0,114 & 0,135 & $-0,154$ & 0,382 \\
\hline
\end{tabular}

estadísticamente significativas en 14 de ellos. Sólo se aprecian diferencias en el rasgo Confiable que aparece con mayor valoración en la UMAG en relación a la UTA. En el caso de la UTA, se observa la presencia de 15 rasgos con medias iguales o superiores a 3,0 con mayor concentración en cuatro de los cinco factores (Competencia, Sinceridad y Familiaridad y Emocionante), con mayor presencia del factor Competencia. Por su parte, la UMAG presenta mayor cantidad de rasgos con medias iguales o superiores a 3,0 con un total de 22 rasgos y con presencia de cuatro de los cinco factores (Competencia, Sinceridad; Familiaridad y Emocionante), lo que denota una personalidad de marca más compleja. La mayor concentración de rasgos con mayores puntuaciones pertenecientes a los factores Competencia y Sinceridad, es una evidencia que la decisión estratégica de centrar los esfuerzos en la calidad, es percibida por los estudiantes, quienes valoran como característicos de la personalidad de marca de sus carreras, a aquellos rasgos asociados y esperados en una formación educacional y universitaria de calidad. Esto se confirma a partir de lo planteado por Ouwersloot \& Tudorica (2001) en relación a que los rasgos de personalidad de marca son formados o influenciados por cualquier contacto directo o indirecto de los usuarios con la marca, por lo que estas características indicarían, desde la percepción de los usuarios, un reflejo del servicio educacional recibido.

Por otra parte, emergen otros rasgos asociados a la percepción de una carrera orientada al contacto interpersonal y a la vinculación afectiva reflejados en el factor Familiaridad que parecen reflejar características de la acogida y el tipo de relaciones interpersonales que los estudiantes perciben al interior de su carrera, probablemente tanto a nivel de las relaciones profesoralumno como del contacto entre pares. Este es un aspecto común a otros estudios en personalidad de marca en carreras de psicología y que probablemente 
refleje aspectos idiosincráticos de formación para la profesión que deberán ser profundizados en estudios posteriores (Denegri et al., 2009). Por otra parte, en ambas instituciones y con una media levemente mayor en el caso de la UMAG, emergen además otros rasgos vinculados a lo emocionante o excitante que puede ser la carrera, lo que puede ser una variable importante en la mantención de la motivación por parte de los estudiantes.

Vinculado al segundo objetivo, se aprecia en ambas instituciones la presencia de rasgos que podrían ser asociados teóricamente a la influencia de las identidades regionales en la valoración de atributos que los estudiantes consideran relevantes. Como se ha establecido teóricamente, la imagen de marca puede ser considerada como parte de la identidad social de los miembros de un grupo, y por ende, de su autoconcepto (Hogg, 1996). Ello es evidente en la presencia de diferencias significativas en los rasgos confiable, fascinante y honesta donde las medias de la UMAG son mayores a las presentadas por la UTA ubicándose todas por sobre el punto medio de la escala. Es interesante observar que por lo menos dos de estos rasgos, a saber, confiable y honesta se corresponden con los valores que los propios magallánicos estiman que forman parte de su identidad (Martinic, 2009; Molina, 2011).Así, como ya habíamos señalado, se observa una personalidad de marca más definida y con mayor cantidad de rasgos característicos en la carrera de psicología de la Universidad de Magallanes, lo que podría vincularse al nivel y profundidad de la identidad magallánica forjada a partir del aislamiento propio de su ubicación geográfica lo que la constituye como un territorio con un núcleo y visión de futuro compartido socialmente a través de los años, ello hace probablemente que la percepción de la universidad que les pertenece sea también más nítida y ello se generalice a todas las carreras que la componen. En contraparte, si bien la carrera de psicología de la Universidad de Tarapacá logra características y rasgos definitorios, estos no alcanzan el mismo nivel de intensidad que en la Universidad de Magallanes, infiriendo el papel que juega una identidad más bien en construcción, afectada por un lado por la conjunción de diversos patrones culturales como por la incipiente división administrativa que incide en una aún naciente diferenciación territorial.

Ante este escenario, los desafíos de ambas carreras, situadas en universidades regionales y en regiones con características identitarias propias se vinculan a la necesidad de lograr una formación que sea sensible a la realidad de su región, con responsabilidad social ante las problemáticas de cada territorio y con una definición identitaria en su personalidad de marca que las distinga como universidades públicas, estatales y regionales donde la competencia en su quehacer y la transparencia de su acción, sin restricción de ninguna naturaleza, como no sean las de orden estrictamente académico, y sin adscribirse a ningún otro marco ideológico o religioso, que no sea el interés general del país y el de satisfacer las necesidades de su desarrollo en el nivel nacional y regional. Ello queda claro en el conjunto de rasgos que los estudiantes atribuyen a sus carreras y que, desde la perspectiva del constructo de personalidad de marca, también constituirían rasgos que ellos también perciben como constitutivos de su propia identidad como psicólogos. Ello porque el supuesto tras la idea de personalidad de marca es que el individuo tiende a seleccionar aquellos rasgos que mejor le representan y con los cuales establece una sintonía de identificación (Aaker, 1997; Denegrí et al., 2009).

A partir de lo expuesto, queda claro que se torna esencial que las instituciones de educación superior de dependencia estatal, si bien ligadas principalmente al ámbito de generación del conocimiento y transmisión e innovación a partir de este, se encarguen de desarrollar métodos eficientes de comunicación de sus características hacia el entorno de manera de mostrar una personalidad de marca clara, definida y distintiva, en donde la relación fluida de la institución con sus usuarios directos e indirectos, tanto actuales como potenciales así como con sus redes le permitan instalar estrategias concretas para el mantenimiento de su presencia y el fortalecimiento de una imagen de marca que les distinga claramente no sólo de sus competidores como en el caso de Psicología UTA sino también refleje su sello de universidades públicas (Piña, 2006; Denegri et al., 2009).

Lo complejo de esta tarea de posicionamiento es que transcurre en un escenario de mercado que crece a un ritmo exponencial, y en el que la oferta de carreras universitarias sobrepasa claramente a la demanda y necesidad país, lo que puede incidir tanto en el nivel de remuneraciones como de seguridad laboral de los futuros profesionales. Esta situación es lo que Baumann (2010) señala como tendencias patológicas del sistema económico y social actual, que 
desembocan en un colapso de la oferta natural y en un exceso de información persuasiva que debilita las capacidades de toma de decisión es de los usuarios. En este escenario, se requiere de una información más focalizada, especifica y clara que recoja las percepciones de los estudiantes y del entorno y que oriente las estrategias de posicionamiento hacia aquellos rasgos diferenciadores que constituyen la esencia de la identidad de las instituciones y de su oferta formativa. Esta sintesis entre la universidad y lo que esperan sus usuarios y las características que en ella proyectan permite entrelazar sus funciones y metas (docencia de calidad, generación de conocimiento e innovación y vinculación con el medio) con las necesidades y expectativas de su entorno regional, lo que a su vez fortalece el vínculo afectivo y cognitivo entre las demandas del medio y la oferta pertinente de las carreras y servicios universitarios (OECD, 2007).

Si bien esta investigación ha abordado a un nivel de relación teórica el vínculo entre la personalidad de marca percibida por los estudiantes y los rasgos identitarios de las regiones donde estas carreras están insertas, resulta necesario abordar esta relación de manera empírica en futuras investigaciones utilizando instrumentos que evalúenla identidad regional y la correlacionen con la personalidad de marca percibida. Ello resulta relevante para el posicionamiento y fortalecimiento de estas instituciones que constituyen claramente una reserva de la tradición de la educación superior pública en Chile y de sus valores.

\section{BIBLIOGRAFÍA}

Aaker, D. (1992). The value of brand equity. Journal of Business Strategy. 13(4), 27-32.

Aaker, J. (1997). Dimensions of Brand Personality. Journal of Marketing Research. 34, 347-356.

Aaker, J., Benet-Martinez, V. \& Garolera, J. (2001). Consumption symbols as carriers of culture: A study of Japanese and Spanish brand personality constructs. Journal of Personality and Social Psychology, 81(3), 492-508.

Álvarez, C. \& Harris, J. (2002). Assesing the structure of brand personality among global and local Mexican brands. AMA Summer Educators Conference, San Diego, Estados Unidos.

Atmann, C.A. (1997). Identidad regional y articulación de los actores sociales en procesos de desarrollo regional. Revista
Austral de Ciencias Sociales, 1, 5-14. Extraído el 20 de Junio de 2011 desde http://mingaonline.uach.cl/scielo. php?pid=S0718-17951997000100001\&script=sci_arttext.

Barrios, M. \& Massa, C. (1999). Dimensiones de la personalidad de marca en Venezuela. Tesis de Maestría, Instituto de Estudios Superiores de Administración.

Bauman, Z. (2010). Mundo Consumo. Ética del individuo en la aldea global. Buenos Aires: Paidós.

Biel, A. (1993). Converting image into equity.Brand Advertising. Hillsdale: Lawrence Erlbaum.

Boisier, S. (2006). La imperiosa necesidad de ser diferente en la globalización: el mercadeo territorial. Territorios $15,71-85$

Brunner, J.J. (2008). El sistema de educación superior en Chile: un enfoque de economía política comparada. Avaliação Campinas 13(2), 451-486.

Chapleo, C. (2005). Do Universities have "succesful" Brands? International Journal of educational advancement, 6(1), 54-59.

Chapleo, C. (2010). ¿What defines "successful" university brands? The International Journal of Public Sector Management, 23(2), 169.

Colmenares, O. \& Saavedra, J. (2008).Dimensiones de personalidad de marca. Caso de estudio: Cadenas de Farmacia. Revista Venezolana de Gerencia, 13(42), 220-232.

CENTRO DE ESTUDIOS REGIONALES CEUTA. (2009). Estudio de opinión pública "Barómetro regional 2009": Análisis Región de Magallanes. Arica: Universidad de Tarapacá. Extraído el 14 de Agosto de 2011 desde http:// www.subdere.gov.cl/sites/default/files/documentos/ region_de_magallanes.pdf.

CONSEJO NACIONAL DE EDUCACIÓN (2010). Índices. Santiago: CNED.

Denegri, M.; Cabezas, D.; Herrera, V.; Páez, A. \& Vargas, M. (2009). Personalidad de marca de carreras de psicología de universidades estatales en Chile: Un estudio descriptivo. Revista IIPSI 12(2), 13-23.

Denegri, M. (2007). Introducción a la Psicología Económica. Colombia: PSICOM Editores.

Diaz, A., Galdames, L \& Ruz, R. (2010) Nación e Identidad en Los Andes. Arica: Ediciones Universidad de Tarapacá.

Díaz, A., Díaz A. \& Pizarro, E. (2010). Arica siglo XX. Historia y Sociedad en el extremo norte de Chile. Arica: Ediciones Universidad de Tarapacá.

Estrada, C. \& Avendaño S. (2008) ¿Consideran los magallánicos que su cultura posee una esencia única? Estudio descriptivo de la aplicación de un instrumento de evaluación de esencialismo cultural a una muestra puntarenense. 
Magallania, 36(2), 155-162.

Freling, T.; Crosno, J. \& Henard, D. (2010). Brand personality appeal: conceptualization and empirical validation. Journal of the Academy of Marketing Science, 39(3), 392-406.

Gonzáles, V. (2002).¿Qué significa ser un profesional competente? Reflexiones desde una perspectiva psicológica. Revista Cubana de Educación Superior, 22(1), 45-53.

Herrera, M. (2011).Intensidad y variables asociadas a la identidad regional de universitarios que estudian en las regiones de Arica y Parinacota, Libertador Bernardo O'Higgins, Los Ríos y en la región de Magallanes y Antártica Chilena. Tesis para optar al Título de Psicóloga. Universidad de Chile. Extraído el 4 de abril de 2012 de www.tesis.uchile. cl/tesis/uchile/2011/.../cs-herrera_m.pdf.

Hogg, M.A. (1996). Social Identity, self-categorization and the small group. En E.H. Witte \& J.D. Davis (Eds.), Understanding group behavior. Mahawah,New Jersey: Lawrence Erlbaum Assiciates, Publishers.

Juliá, M. (2006).Competencias profesionales del psicólogo educacional: una tarea asociativa. Revista de Psicología, 15(2), 115-130.

Keller, K. (1993). Conceptualizing, measuring, and managing customer-based brand equity. Journal of Marketing, 57(1), 1-22.

Koebel, M. \& Ladwein, R. (1999). L'échelle de personnalité de la marque de Jennifer Aaker: Adaptation au contextefrançais. Decisions Marketing, 18, 81-88.

Lane, K. \& Richey, K. (2006) The importance of corporate brand personality traits. Brand Management, 14(1/2), 74-81.

Larrain, J. (2005). Integración regional e identidad nacional: Chile ¿país modelo? Revista del Sur, 161, 7-15.

Martinez, J. (2008). Perfil de valor de marca y la medición de sus componentes. Academia Revista Latinoamericana de Escuelas de Administración, 41, 69-89.

Martinic, M. (2009). Región Magallánica: una identidad bien definida con sus expresiones territoriales. Extraído el 1 de agosto de 2010, desde http://www.umag.cl/construyendoregion/articulo.php?idarticulo $=34$.

Méndez, M. L. (2009). Proceso de descentralización e identidad regional: ¿Cómo se perciben los habitantes de regiones y cómo perciben los procesos de desarrollo regional? Proyecto Desigualdades (Anillos SOC12). Santiago: Universidad de Chile. Extraído el 1 de Septiembre 47 de 2011 desde http://www.desigualdades.cl/wp-Content/ uploads/2010/03/Informe identidad regional Mendez_octubre 2009.pdf.

Mercado, V. (2004). Ranking general de marcas chilenas. Tesis para optar al título de Ingeniero Comercial, Universidad de Chile.
Molina, W. (2011). Identidad regional en Magallanes, sus expresiones simbólicas y territoriales. Magallania, 39(1):59-69.

PORTAL MI FUTURO. CARRERA DE PSICOLOGIA. Extraído el 3 de abril de 2013. http://www.mifuturo.cl/index.php/ donde-y-que-estudiar/buscador-de-carreras.

OECD (2007). La educación superior y las regiones globalmente competitivas y localmente comprometidas. Francia: OECD Publications.

Ouwersloot, H. \& Tudorica, A. (2001). Brand personality creation through advertising. Maastricht: Maastricht University, Maastricht Academic Center for Research in Services.

Parker, A., Neary, M. \& Lambert, C. (2005).Reino Unido: la educación superior en la edad moderna. Calidad en la Educación, 23(2), 319-334.

Piña, I. (2006). La universidad en el siglo XXI. Revista de la Educación Superior,35, 115-122.

Pirela, L.; Villavicencio, H. \& Saavedra, J. (2004). Dimensiones de personalidad de marca. Estudio exploratorio en Venezuela. Revista de Ciencias Sociales 10(3), 430-440.

Plummer, J. (1985). How does personality makes a difference. Journal of Advertising Research 21, 27-31.

Podesta, J. (2004). Claves para entender el desarrollo de la Región de Tarapacá. Revista de Ciencias Sociales, 14, 20-35.

PORTAL DE CARRERAS UNIVERSITARIAS (2013) En http:// universia. carreras.universia.cl.

Semprini, A. (1995). El marketing de la marca, una aproximación semiótica. Barcelona: Ediciones Paidós.

Saavedra, J., Colmenares, O. \& Pirela, J. (2007). Correlación entre dimensiones de personalidad de marca y la marca emocional. Estudio en Cadena de Farmacias. Revista de Ciencias Sociales 13(2), 219-229.

Saavedra, J., Urdeneta, D., Pirela, J. \& Colmenares, O. (2008). Medición de la personalidad de marca en el mercado automotriz. Visión Gerencial, 7(1), 183-196.

Sedikides, C. \& Brewer, M. (2001). Individual self, relational self, collective self. Philadelphia: Psychology Press.

Siguaw, J., Mattila, A. \& Austin, J. (1999). The Brand-Personality Scale. An Application for Restaurants. Cornell Hotel and Restaurant Administration Quarterly, 40(3), 48-55.

SUBSECRETARÍA DE DESARROLLO REGIONAL Y ADMINISTRATIVO (2009). Identidad regional. Reconociendo la diversidad para el desarrollo de los territorios. Santiago: SUBDERE.

SUBSECRETARÍA DE DESARROLLO REGIONAL Y ADMINISTRATIVO (2010) Identidad Regional y Desarrollo para Magallanes. Santiago: SUBDERE.

Suppehellen, M. \& Gornhaug, K. (2003). Building foreign brand personalities in Russia: the moderating effect of consumer ethnocentrism. International Journal of 
Advertising, 22(2), 203-226.

Yongjun, S. \& Jooyoung, K. (2010). Effects of Brand Personality on Brand Trust and Brand Affect. Psychology \& Marketing, 27(7), 639-661.

Zúñiga, C. \& R. Asún (2003). 'Identidad regional en un contexto de cambio. Un estudio en la Araucanía, Chile', Psicología Política 26,73-92. 
M. DENEGRI et al. 